\title{
Management von Künstlicher Intelligenz in Unternehmen
}

\author{
Benjamin van Giffen* \\ University of St.Gallen \\ Institute of Information Management \\ St.Gallen, Switzerland \\ benjamin.vangiffen@unisg.ch
}

\author{
Damian Borth \\ University of St.Gallen \\ Institute of Computer Science \\ St.Gallen, Switzerland \\ damian.borth@unisg.ch
}

\author{
Walter Brenner \\ University of St.Gallen \\ Institute of Information Management \\ St.Gallen, Switzerland \\ walter.brenner@unisg.ch \\ * corresponding author
}

Zusammenfassung: Künstliche Intelligenz bietet Unternehmen neue Möglichkeiten Prozesse, Produkte, Dienstleistungen und Geschäftsmodelle zu innovieren und bestehende zu verändern. Daher wird das professionelle Management Künstlicher Intelligenz in Unternehmen zu einer zentralen Aufgabe, um die neuen Wertversprechen mit produktiven Systemen zu realisieren.

Der Beitrag stellt das St.Galler Management Modell für KI (SGMM-KI) vor und zeigt sieben Handlungfelder für den betrieblichen Einsatz von KI: (1) Management von Künstlicher Intelligenz, (2) Organisation des Betriebs, (3) Rechtliche Gestaltung, (4) Regulierung und Compliance, (5) Lebenszyklus-Management, (6) Management der Technologie-Infrastruktur, sowie (7) Cybersicherheit.

Der vorliegende Artikel leitet konkrete erste Schritte an und richtet sich primär an Geschäftsleitungsmitglieder, IT- und Innovationsverantwortliche sowie Projektleiter, welche die neuen Wertversprechen der KI in der betrieblichen Praxis verwirklichen möchten.

Schlüsselwörter: Künstliche Intelligenz, Führung, Management, Management System, Modell

\section{Reference:}

van Giffen, B., Borth, D., \& Brenner, W. (2020). Management von Künstlicher Intelligenz in Unternehmen. HMD Praxis der Wirtschaftsinformatik, 57(1), 4-20. 


\section{Managing Artificial Intelligence in Business Organizations}

Abstract: Artificial intelligence offers firms new opportunities to innovate processes, products, services and business models and to change existing ones. Therefore, the professional management of artificial intelligence in companies becomes an increasingly important task to realize the new value propositions with productive systems.

The article presents the St.Gallen Management Model for AI (SGMM-AI) and highlights seven areas of action for the operational use of AI: (1) management of artificial intelligence, (2) organization of business operations, (3) legal, (4) regulation and compliance, (5) life-cycle management, (6) management of technology infrastructure, and (7) cyber security.

This article provides guidance on the first steps of implementing AI. It is primarily targeted board members, IT, innovation and project managers who want to put the new value propositions of AI into practice.

Keywords: artificial intelligence, leadership, management, management system, model 


\section{Management von Künstlicher Intelligenz in Unternehmen}

\section{Künstliche Intelligenz als neue Technologie in Unternehmen}

\subsection{Künstliche Intelligenz ist ein aktuelles und wichtiges Thema für viele Unternehmen}

Künstliche Intelligenz (KI) bietet Unternehmen neue Möglichkeiten Prozesse, Produkte, Dienstleistungen und Geschäftsmodelle zu innovieren und bestehende zu verändern. Unternehmen sämtlicher Branchen investieren derzeit in den Aufbau von KI-Fähigkeiten. Viele der der grossen Technologieunternehmen wie zum Beispiel Google, Facebook, Alibaba, oder auch Amazon richten Ihre Unternehmensstrategie und Investitionsplanung auf den Einsatz von KI aus.

Künstliche Intelligenz ist in Wissenschaft und Praxis ein allgegenwärtiges Thema. Die zunehmende Digitalisierung trägt hierzu bei und hat in der Vergangenheit bereits den Aufbau von Data-Analytics Teams und Data Lakes als Grundlage für die Arbeit mit KI sowie der Entwicklung erster KI-Prototypen geführt. Eines ist klar: Unternehmen wollen KI in der betrieblichen Wertschöpfung einsetzen.

KI schafft aber nicht nur einzigartige Möglichkeiten mit Technologie Wertpotentiale zu realisieren, KI erfordert auch ein Um- bzw. Neudenken darüber wie Unternehmen Technologie einsetzen können. Dieses Umdenken folgt nicht nur aus technologischen Besonderheiten, sondern auch aus der (teilweise) begründeten Wahrnehmung von Risiken und Bedrohungen, die im öffentlichen Diskurs mit dem Einsatz von KI verbunden werden. Demensprechend wird der Einsatz von KI im betrieblichen Umfeld gekennzeichnet durch eine Vielzahl neuer Phänomene, die nachfolgend vorgestellt werden und damit den Rahmen für das im Folgenden vorgestellte Managementmodell für den betrieblichen Einsatz von KI bilden.

\subsection{Künstliche Intelligenz liefert neue Wertversprechen für Unternehmen}

KI ermöglicht die Entwicklung neuer Anwendungen, die sich essentiell von traditionellen Software-Lösungen unterscheiden. Im Alltag werden zunehmend Verfahren der KI und des maschinellen Lernens (eng. Machine Learning, ML) verwendet, zum Beispiel, um natürliche Sprache zu verarbeiten oder Objekte in Bildern zu erkennen. Die Vielzahl an KI-Technologien ermöglicht zum Beispiel sprachgesteuerte Interaktionen mit digitalen Assistenten, das Durchsuchen grosser Mengen an Bildern nach bestimmten Objekten oder Personen, oder auch das Erkennen von Anomalien in Finanztransaktions- oder Netzwerkdatenströmen.

Die «Datafizierung» sämtlicher unternehmerischer Prozesse und Aktivitäten sowie permanent verfügbare, leistungsfähige und nahezu unbegrenzt skalierbare Rechenkapazitäten schaffen Rahmenbedingungen, die grössere Investitionen in Forschung und Entwicklung KI-gestützter Produkte und Dienstleistungen für Unternehmen zunehmend wertvoller machen (von Krogh, 
2018). Dementsprechend hat Künstliche Intelligenz das Potential, unsere bisherige Art zu leben und zu arbeiten, grundlegend zu verändern.

\subsection{Künstliche Intelligenz erfordert einen Paradigmenwechsel in der Entwicklung}

Die KI-Systementwicklung unterscheidet sich grundsätzlich von der traditionellen Softwareentwicklung. Das zentrale Paradigma der KI-Entwicklung lautet „Trainieren statt Programmieren“. Im Gegensatz zur Softwareentwicklung erfordern Verfahren der Künstlichen Intelligenz, insbesondere das Trainieren tiefer Neuronaler Netze die Verarbeitung großer Mengen an Trainingsdaten, um die begehrten Neuronalen Netze zu parametrisieren. Infolgedessen ist extreme Rechenleistung vor allem für die Entwicklung von KI-Modellen erforderlich und viel weniger im Rahmen des "normalen" Geschäftsbetriebs. Software hingegen benötigt in der Entwicklungsphase tendenziell deutlich weniger Rechenleistung als im späteren betrieblichen Einsatz.

Auch die Prozesse zur Generierung von KI-Modellen unterscheiden sich von der Programmierung traditioneller Softwareanwendungen. Softwareanwendungen basieren auf einer Codebasis, die vorhersehbar und deterministisch auf einem Computer läuft, während KIModelle aus der algorithmischen Verarbeitung von Trainingsdaten resultieren. Die von KIModellen erzeugten Vorhersagen basieren dann auf dem vorhergenden Training des jeweiligen KI-Modells.

Insofern liefern traditionelle IT-Projekte typischerweise eine Softwareanwendung, die durch das Schreiben und Kompilieren eines Quellcodes erzeugt wird. Der Softwareentwicklungsprozess folgt üblicherweise einem der etablierten Prozessmodell, wie z.B. das V-Modell oder agilen Methoden wie Scrum, Extreme Programming oder Ähnlichem (Conboy, 2009; Wang, Conboy, \& Pikkarainen, 2012), wodurch ein weitgehend standardisierter und vorhersehbarer Ablauf gewährleistet wird.

KI-Projekte hingegen nutzen Verfahren des maschinellen Lernens um KI-Modelle zu trainieren (LeCun, Bengio, \& Hinton, 2015). In diesem Zusammenhang bezeichnet Training den Prozess der Extraktion von in Daten gespeichertem Wissen durch die Anwendung von maschinellen Lernalgorithmen. Der Trainingsprozess selbst ist ein iterativer, hochautomatisierter und rechenintensiver Prozess, bei dem das resultierende KI-Modell maßgeblich durch den eingesetzten Lernalgorithmus, dessen Parametrisierung und die während des Trainings verarbeiteten Daten beeinflusst wird.

\subsection{Künstliche Intelligenz schafft neue Bedrohungsszenarien und Cybersicherheits- risiken}

In Zukunft werden KI-Systeme zunehmend eingesetzt werden, um Entscheidungsprozesse zu automatisieren. Hierfür interagieren KI-Systeme mit ihrer Umwelt, indem sie Input Daten (z.B. Bilder, Texte, Ton) mithilfe eines KI-Modells verarbeiten und entsprechende Ausgaben, 
Vorhersagen oder Entscheidungen automatisiert ausgeben (z.B. in Bildern, Texten, Ton oder anderen Output Daten). Hierbei entstehen neue Cybersicherheits- und Bedrohungsszenarien, die sich aus Manipulationen an der Datenschnittstelle von KI-Modellen ergeben, da KIgestützte, automatisierte Entscheidungssysteme empfindlich gegenüber dem gegebenen InputDatenstrom sind. Angreifer könne diese Eigenschaft ausnutzen, indem sie Eingabedaten manipulieren, um auf diesem Weg dann ein bestimmtes (gewünschtes) Verhalten des KISystems auslösen.

Ein kürzlich bekanntgewordenes Beispiel für eine solche Manipulation an der Datenschnittstelle war das Manipulieren des Lenkverhaltens eines autonomen Fahrzeugs. Forscher hatten im Modellversuch Aufkleber auf einer Strasse platziert und dem Autopiloten des Fahrzeugs vorgespielt, die Fahrspur würde sich verschieben. In der Folge lenkte das Fahrzeug in die entsprechende Richtung (Goodin, 2019). Eine grosse Herausforderung bzw. Risiko in solchen Systemen entsteht also an den Datenschnittstellen der KI, welche häufig ungeschützt gegen solche manipulativen Angriffe sind.

Ein weiteres, ausgefeiltes Bedrohungsszenario ergibt sich aus sogenannten generativen feindlichen Netzwerken (eng. Generative Adversarial Networks, GANs), welche durch systematisches, kontinuierliches Testen eines Neuronalen Netzes dessen Entscheidungsverhalten erlernt und anschliessend die gezielte Manipulation bzw. Generierung von Eingabedaten ermöglicht, um gewünschte Ausgaben bzw. Entscheidungen herbeizuführen (diese werden auch Generative Neuronale Netze oder im Falle einer bösartigen Absicht auch Generative Adversarial Neuronal Networks genannt (Goodfellow, Shlens, \& Szegedy, 2014). Ein weiteres prominentes Beispiel für ein generatives Netz liefert das sogenannte „Deep Fake Obama Video“. Das Video zeigt wie dem ehemaligen amerikanischen Präsidenten beliebige Botschaften in den Mund gelegt werden können, in dem die entsprechenden Bild- und Sprachsequenzen in ein Video synthetisiert werden.

\subsection{Künstliche Intelligenz wird von Kunden und Mitarbeitern kritisch wahrgenommen}

Neu am Einsatz von KI sind nicht nur die Anwendungsgebiete, Entwicklungsprozesse und technologiebedingten Bedrohungsszenarien, sondern auch die kritische Wahrnehmung von KITechnologie durch Mitarbeiter und Kunden. In der öffentlichen Diskussion polarisiert KI in zwei Lager: Die eine Gruppe preist KI als heilsbringende Technologie, die in naher Zukunft sämtliche unternehmerischen und zivilisatorischen Probleme lösen wird. Die andere Gruppe lehnt KI ab und verweist auf eher apokalyptische Situationen, in denen autonome Roboter und Systeme Fachkräfte ersetzen oder gleich beherrschen werden. Die Fantasie der Beteiligten Diskutanten scheint hierbei keine Grenzen zu kennen; der aktuelle Einsatz von KI sollte jedoch deutlich differenzierter betrachtet werden. 
Heutige Nutzungsszenarien der KI sind meist sehr eng auf spezifische Aufgaben begrenzt (enge KI), so dass von einer allgemeinen, die menschlichen Fähigkeiten übersteigende KI (weite KI) sicher noch nicht die Rede sein kann. Im Hinblick auf den betrieblichen, professionellen Einsatz von KI lohnt es sich allerdings darüber nachzudenken, warum KI so viel kritischer als andere Technologien wahrgenommen wird:

Erstens ist KI und hierbei insbesondere tiefe Neuronale Netze mit ihren vielen Schichten im Allgemeinen (noch) eine Black-Box-Technologie. KI kann zwar hervorragend Datenobjekte klassifizieren, die getroffenen Vorhersagen und Entscheidungen sind für den Menschen aber aufgrund der Komplexität der zugrundeliegenden Modelle nicht oder nur kaum nachvollziehbar.

Zweitens können durch die Auswahl und Akquisition von Trainingsdaten sogenannte datenbasierte Verzerrungen (eng. Data Bias) eingeführt werden. In der Vergangenheit hat das unzureichende Management von datenbasierten Verzerrungen zu KI-Modellen geführt, die z.B. in der Bilderkennung ethnische Minderheiten diskriminiert haben (Zhang, 2015) oder Frauen in der Auswahl von Bewerbenden auf eine Stelle herausgefiltert haben (Reuters, 2018). Solche moralisch bedenklichen, teilweise auch verwerflichen Anwendungen bergen dementsprechende Reputationsrisiken und werden regelmässig durch Journalisten, die Politik und auch einzelne Kunden und Mitarbeiter aufgegriffen und medial verwertet.

Drittens haben viele Menschen konkrete Sorgen und Ängste im Zusammenhang mit KI und zwar sowohl auf individueller (z.B. Arbeitsplatzverlust, Verlust von Datenschutz oder Privatsphäre), als auch organisationaler Ebene (z.B. Gefährdung der Integrität des Unternehmens, Untergrabung des Entscheidungsverhaltens, fehlende Nachvollziehbarkeit von KI-gestützten Entscheidungen).

Vor diesem Hintergrund ist Vertrauen in KI-Technologie und KI-Systeme nicht nur ein gesellschaftliches, sondern ganz besonders auch ein unternehmerisches Thema. Der betriebliche Einsatz von KI-Systemen kann sehr leicht - auch unbeabsichtigt - Kritik und Widerstände innerhalb und ausserhalb des Unternehmens hervorrufen. Dementsprechend gilt es für Entscheider und Beteiligte ethische, rechtliche und sozial akzeptierte Praktiken für den Einsatz von KI-Systemen zu implementieren. Grundlage für eine solche „Vertrauenswürdige

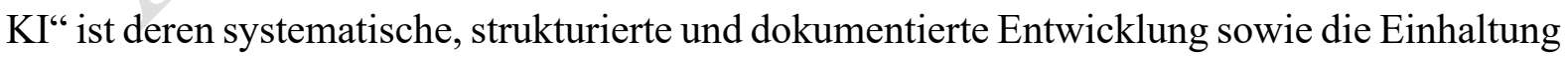
geltender technischer und nicht-technischer Normen und Prinzipien (European Commission, 2019).

\subsection{Zielsetzung des Artikels}

Folgt man den obigen Ausführungen zur KI im betrieblichen Umfeld wird unmittelbar klar, dass KI eine Vielzahl neuer Chancen bietet, aber auch neue Problemstellungen aufwirft für die es noch keine Standardlösungen gibt. Für Unternehmen und Führungskräfte ist es jedoch nicht 
immer einfach, die mit Auswahl, Einsatz und dem Betrieb von Lösungen im Kontext der KI notwendigen Managementfragen zu stellen und angemessen zu beantworten.

Vor diesem Hintergrund soll der vorliegende Artikel eine Hilfestellung bieten, wie die Umsetzung theoretischer Ansätze und technologischer Konzepte in die Praxis gelingen kann. Das Zielbild ist es dabei jedoch nicht, den Leser zum KI-Spezialisten auszubilden, sondern vielmehr ein persönliches Verständnis zu erarbeiten, mit dem Einsatzpotentiale abgeschätzt, Investitionsentscheidungen überblickt und die strategische Tragweite solcher Entscheidungen abgeschätzt werden kann.

\section{Das Management von KI ist eine Führungsaufgabe}

Viele Grossunternehmen kommunizieren nahezu täglich ihre ambitionierten Pläne zur Nutzung von KI. Ein Beispiel: «Binnen drei Jahren will Bosch-Chef Volkmar Denner die Zahl der KIExperten auf 4000 im Konzern vervierfachen» (Handelsblatt, 2019). Unabhängig davon, ob es Unternehmen der traditionellen Industrien gelingt die Rekrutierung von KI-Experten im angekündigten Umfang zu bewältigen, stehen nachgelagerte, wichtige Fragestellungen an. Der betriebliche, produktive Einsatz von $\mathrm{KI}$ ist keinesfalls eine Disziplin nur für einzelne Spezialisten. Der Einsatz von KI in marktreifen Lösungen im industriellen Maßstab wird die gesamte Organisation benötigen. Er gleicht einem Zehnkampf, bei dem unterschiedliche Kompetenzen gefragt sind. Zum Beispiel Domänenwissen, um Einsatzpotentiale und Use Cases zu identifizieren, die Fähigkeit KI-Algorithmen einzusetzen, aber auch die technologische Kompetenz, um Kunden KI-gesteuerte Produkte und Dienste erklären zu können. Folglich wird der KI-Einsatz in der Breite eine Vielzahl von Unternehmensbereichen und -funktionen tangieren. Das Management von KI betrifft nicht nur die Bereiche Forschung und Entwicklung, sondern fordert insbesondere auch die Unternehmensführung sowie das ITManagement in der Bereitstellung entsprechender KI-bezogener Dienstleistungen. Deshalb ist auch schon heute klar, dass das Management von KI eine strategische Kompetenz für Unternehmen und insbesondere CIOs ist.

KI liefert eine Fülle neuer Herausforderungen und Fragestellungen, welche viele Unternehmen noch nicht für sich beantwortet und oftmals noch nicht erkannt haben. Die ausgeführten Merkmale von KI-Technologie haben Implikationen für klassischer Managementfragen wie zum Beispiel:

- Welche Implikationen hat KI innerhalb unserer Strategie? Wie können KI-Projekte sicher und effizient durchgeführt werden? Welche Voraussetzungen müssen geschaffen werden?

- Welche Fähigkeiten und welche Organisationstruktur brauchen wir, um die Potentiale von KI voll auszuschöpfen?

- Welche rechtlichen, regulatorischen und vertraglichen Grundlagen müssen wir in der produktiven Nutzung von KI berücksichtigen? 
- Wie könnte ein KI-Lebenszyklus-Management aussehen, dass sich gut in unsere bestehenden Prozesse einfügt?

- Wie müssen Prozesse für Auswahl, Implementierung und Betrieb einer KI-TechnologieInfrastruktur definiert sein?

- Welche Massnahmen sind geeignet Cybersicherheit herzustellen und gegnerischen Angriffe auf KI-Systeme effizient abzuwehren?

Die beschriebene Ausgangslage macht deutlich, dass für Unternehmen und insbesondere auch IT-Organisationen Handlungsbedarf in der Entwicklung von KI-Fähigkeiten besteht. Die anstehenden Probleme sind neuartig und es gibt nur wenig verfügbares Anwendungswissen. Es ist klar, dass es hierfür keine „One size fits all“ Lösung oder ein allgemeingültiges Referenzmodell geben wird, sondern dass sich jede Organisation selbst überlegen muss wie der Einsatz von KI in ihrem betrieblichen Kontext gelingen kann.

Der erste Schritt dieser Klärung besteht jedoch nicht in der Herleitung theoretischer Referenzprozesse oder Architekturen, sondern vielmehr darin die wichtigsten Themen und Handlungsfelder zu erkennen und die richtigen Fragen zu stellen, um erste Projekte durchzuführen und initiale Leitlinien für den Einsatz von KI im Unternehmen zu entwickeln. Darauf aufbauend und basierend auf einem tiefgreifenden Verständnis der Technologie können dann Managementimplikationen abgeleitet werden und diese schrittweise in eine systematisches Managementmodell überführt werden.

In vielen anderen Bereichen wurde der professionelle Einsatz neuer Technologien und Vorgehensweisen durch Managementmodelle bereits erfolgreich definiert. Zum Beispiel werden Managementmodelle im Projektmanagement (vgl. PMI/PMBoK, PRINCE2), im ITService Management (vgl. ITIL), in der IT-Governance (vgl. COBIT) oder auch dem Data Mining (vgl. Crisp-DM) selbstverständlich verwendet, um systematische und einheitliche Vorgehen in der Umsetzung bestimmter Aufgaben zu verwirklichen.

Ein Managementsystem für KI hilft bei der Identifikation und Realisierung neuer Wertversprechen in dem es einen Ordnungsrahmen bietet, um die Vielzahl an Aufgaben, die gleichzeitig bearbeitet und gemanagt werden müssen, zu überblicken und das Ergebnis zielgerichtet zu gestalten. Mit anderen Worten: Ein Managementmodell zeigt Handlungsfelder und deren Zusammenhänge systematisch auf und hilft diese zu bearbeiten.

\section{Das St.Galler Management Modell für den betrieblichen Einsatz von KI}

Das hier vorgestellte St.Galler Management Modell für KI (SGMM-KI) entsteht aus der Forschungsgruppe „Management von Künstlicher Intelligenz“, einer Kooperation des Instituts für Wirtschaftsinformatik und des Instituts für Informatik der Universität St.Gallen (vgl. Abb. 1). Zahlreiche Interviews, Workshops und Gespräche mit Fach- und Führungsverantwortlichen 
in der IT, sowie CIOs, CDOs sowie Data Science- und Analytics-Verantwortlichen dienten als empirische Grundlage für diese erste Version.

Die mittelfristige Zielsetzung des Modells besteht darin, basierend auf einem tiefen technischen Verständnis KI-Projekte und KI-Prototypen analysieren zu können und Einflüsse auf das Managementsystem abzuleiten. Ausgehend von einer technischen Sicht auf heutige und zukünftige KI-Systeme werden die identifizierten Implikationen in eine systematische Managementsicht überführt. Langfristig können dadurch geeignete Prozesse, Strukturen, Rollen, KPIs und Dokumente erarbeitet werden, mit der KI im betrieblichen Kontext erfolgreich gemanagt werden kann. Wir erwarten, dass sich mit Einführung und Betrieb von KI-Systemen bestehende Konzepte wie z.B. Berechnung von Business Cases, Total Cost of Ownership, oder Return-on-Investment entweder neu gestaltet, mindestens aber angepasst werden müssen, um der Neu- und Andersartigkeit von KI-Systemen Rechnung zu tragen.

Management von Künstlicher Intelligenz (KI)

(1) Initiale KI-Projekte durchführen, (2) Organisationales Lemen sicherstellen

Organisation
des Betriebs
(1) Identifikation von
KI-Potentialen in
Business und Use
Cases
(2) Aufbau und
Entwicklung von KI-
Fähigkeiten in der
Organisation
(3) Gestaltung des
Anbietermanagements
für KI-Dienste

Rechtliche
Gestaltung
(1) Sicherung von
geistigem Eigentum an
KI-Modellen
(2) Gestaltung
haftungsrechtlicher
Fragestellungen

Regulierung \&
Compliance
(1) Identifikation und
Übersetzung der
anwendbaren
regulatorischen
Anforderungen
(2) Implementierung
von Governance, um
Regularien
umzusetzen

Lebenszyklus-
Management
(1) Management von
Trainingsdaten und
Data Bias
(2) Versionierung und
Versionsmanagement
von KI-Modellen

Technologie-
Infrastrukur
(1) Auswahl
geeigneter KI-
Frameworks, KI-
Bibliotheken und KI-
Plattformen
(2) Weiterentwicklung
bestehender IT-
Infrastrukturprozesse

Cybersicherheit

(1) Identifikation von Angriffspunkten auf eingesetzte KISysteme

(2) Bewusstsein für neue KI-Bedrohungsszenarian schaffen

\section{Abb1.: Das St.Galler Management Modell} für den betrieblichen Einsatz von Künstlicher Intelligenz

Das St.Galler Management Modell für KI unterscheidet sieben Handlungfelder für den betrieblichen Einsatz von KI: (1) Management von Künstlicher Intelligenz, (2) Organisation des Betriebs, (3) Rechtliche Gestaltung, (4) Regulierung und Compliance, (5) LebenszyklusManagement, (6) Management der Technologie-Infrastruktur, sowie (7) Cybersicherheit. Die sieben Handlungsfelder spiegeln die unterschiedlichen Perspektiven wieder, welche in dieser oder ähnlicher Form häufig in der Organisationsstruktur von Unternehmen verankert sind.

Das Modell liefert also einen Entwurf, mit dem relevante, neue Problemstellungen in der betrieblichen Nutzung von KI strukturieriert und in handhabbare Teilaufgaben zerlegt werden können. Im Folgenden werden die einzelnen Handlungsfelder aufgezeigt und einige der darin enthaltenen Aufgaben näher erläutert. Zu diesem Zeitpunkt besteht die Zielsetzung jedoch noch nicht darin alle Aspekte vollständig zu diskutieren, sondern die in der empirischen Arbeit 
erkannten Herausforderungen darzustellen und der interessierten Leserschaft als Anstoss für das persönliche und organisationale Lernen zu dienen.

\section{Handlungsfelder im betrieblichen Einsatz von KI}

\subsection{Management von KI}

Das Management von KI befasst sich mit der Definition von Organisationsstrukturen und Managementprozessen um KI-bezogene Zielesetzungen aus der Unternehmens- bzw. ITStrategie umzusetzen. Dabei handelt es sich um eine Führungsaufgabe, die das Ziel verfolgt die Organisation zu befähigen KI-Systeme einzuführen und zu produktiv zu betreiben.

Als übergeordnete Aufgabe steuert das Management von KI die zielgerichtete Einbringung von KI-Technologien in die Prozesse und Aktivitäten der betrieblichen Leistungserstellung, wobei die institutionellen und technologischen Rahmenbedingungen explizit berücksichtigt werden. Zentral für diese Aufgabe sind hierfür ein KI-orientiertes Portfoliomanagement sowie die Fähigkeit KI-Systeme mit Methoden des Projektmanagements umzusetzen.

Die meisten Unternehmen haben heute jedoch noch keine definierten Vorgehensweisen und Organisationsstrukturen um KI standardisiert zum Einsatz zu bringen. Vor diesem Hintergrund besteht die erste Aufgabe also nicht darin das Unternehmen zu reorganisieren oder Prozesse zu definieren, sondern in der Schaffung geeigneter Rahmenbedingungen für den mittelfristigen produktiven Einsatz von KI in Produkten, Dienstleistungen oder neuen Geschäftsmodellen.

Die wichtigste Massnahme für den systematischen Aufbau von KI-Fähigkeiten im Unternehmen ist es initiale KI-Projekte durchzuführen und zu managen. Dabei ist nicht nur das produzierte Ergebnis, z.B. die Entwicklung eines Chatbots, wichtig, sondern auch die Durchführung des Projekts an sich sowie die einzelnen Schritte in der Entwicklung des zugrundeliegenden KI-Modells als eine Massnahme des organisationalen Lernens und Reflektierens $\mathrm{zu}$ begreifen. Die Zielsetzung besteht also nicht allein darin, dass im Unternehmen ein vorzeigbarer Prototyp entwickelt, sondern auch dass das Projekt überhaupt durchgeführt wird, um Aufmerksamkeit und Interesse zu generieren, um Mitarbeiter zu ermutigen KI als Lösungsinstrument für betriebliche Problemstellungen zu verstehen und schliesslich um transferierbares Wissen über die Implementierung von KI aufzubauen, damit die organisationalen Fähigkeiten und Kompetenzen schrittweise entwickelt werden können.

Diese projektorientierte Herangehensweise schafft die Grundlage für die systematische, strukturierte und wiederholbare Bearbeitung von KI-Projekten welche einen wichtigen Erfolgsfaktor für die Implementierung von KI-Lösungen im betrieblichen Umfeld darstellt. KIModelle sind keine Einzellösungen, sondern müssen regelmässg in mehr oder weniger komplexe Applikationsumgebungen eingebunden werden. Deshalb ist es auch notwendig die Besonderheiten von KI-Projekten herauszuarbeiten, die sich auch aus den Eigenschaften von KI-Technologien ergeben (z.B. Trainieren statt Programmieren sowie diese als kontinuierlich 
Lernende KI-Modelle zu verstehen). Die zentrale Aufgabenstellungen für die Erarbeitung eines professionellen und für die Organisation passenden KI Projektmanagement-Ansatzes sind daher:

- Die Identifikation von guten, schlechten und nützlichen Praktiken, z.B. für die Akquisition und Erschliessung von Trainingsdaten.

- Die Fähigkeit geschäftliche Problemstellungen und Opportunitäten in ihren jeweiligen Kontext als adäquate KI- bzw. Machine-Learning-Problemstellung zu formulieren.

- Eine "Wertschöpfungsmechanik der KI" zu etablieren, welche die einzelnen Schritte einer KI-gestützten Informationsverarbeitung (z.B. Input/Sensor, handelnder KI-Agent, Output/Steuerung) aufzeigt und auch in der Breite der Belegschaft verstanden wird.

- KI Projekte vertrauensfördernd und überzeugend zu kommunizieren, um die Bedeutung des Themas für die Organisation zu signalisieren.

\subsection{Organisation des Geschäftsbetriebs}

Dieses Handlungsfeld befasst sich mit den operativen Fragestellungen, welche für die betriebliche, produktive Nutzung der KI relevant sind. Hierzu zählen vor allem drei Aufgaben: Die Identifikation von KI-bezogenen Wertschöpfungspotentialen, der Aufbau von KIFähigkeiten in der Belegschaft, sowie die Gestaltung des Sourcingprozesses für KI-bezogene Dienste externer Anbieter.

Erstens, die systematische Identifikation von KI-Wertschöpfungspotentialen anhand von Business und Use Cases. Hierfür gilt es Suchstrategien zu entwickeln, welche Mitarbeiter, insbesondere solche mit tiefem Ablauf- und Domänenverständnis befähigen, betriebliche Aufgaben und Problemstellungen in der Anwendungslogik einer KI zu beschreiben. Zum Beispiel sollten Mitarbeiter Überlegungen einbringen können, welche Prozesse und Aktivitäten mit Verfahren der KI unterstützt oder ersetzt werden könnten. Das Ziel ist hierbei, involvierte Mitarbeiter auszubilden und zu sensibilisieren, zum anderen aber auch zu lernen welche Faktoren im Hinblick auf Anwendbarkeit, Akzeptanz und Nützlichkeit von KI im Kontext der betrieblichen Aktivitäten besonders förderlich sind.

Zweitens, Aufbau und Entwicklung von KI-Fähigkeiten innerhalb der Organisation. KIFähigkeiten, insbesondere in den Bereichen Data Science und Machine Learning, können am externen Markt besorgt werden. Dies geschieht z.B. durch Beratungsleistungen, Crowdsourcing oder die Zusammenarbeit mit technischen Lösungsanbietern, Startups oder Universitäten. Die Vorteile dieses Ansatzes sind u.a. schnelle Bereitstellung der erforderlichen Kompetenzen, sowie die Vermeidung dauerhaft anfallender Personalkosten. Die Nachteile dieses Ansatzes sind jedoch zum einen die Knappheit der eingekauften Spezialisten und damit eng verbunden auch die fehlende Kontinuität auf deren Knowhow zugreifen zu können. Zum anderen erfordert der betriebliche Einsatz von KI, wie eingangs dargestellt, die Einbindung unterschiedlicher Kompetenzen und Wissensträger, da die Entwicklung von KI-Modellen 
immer auch im Kontext und der Domäne des jeweiligen Unternehmens stattfindet. Es ist unmittelbar klar, dass Unternehmen, welche KI ernsthaft einsetzen wollen, nicht darum herumkommen, eigene Fähigkeiten in den unterschiedlichen geforderten Bereichen aufzubauen.

Drittens, Gestaltung des Anbietermanagements für KI-bezogene Dienste. Viele der heute relevanten KI-Infrastrukturen stammen von grossen Technologieanbietern wie z.B. Google Cloud Plattform (GCP) oder Amazon Web Services (AWS). Daneben arbeiten Unternehmen häufig mit spezialisierten Anbietern oder auch Startups zusammen, um konkrete Use Cases gemeinsam umzusetzen. Hierbei stellen sich regelmässig Fragen darüber, wie die eigentliche Leistungserbringung gemessen werden soll, wenn es z.B. eine Vielzahl kostenfrei nutzbarer Alternativen gibt, die auf einer hohen Datenqualität von kuratierten Beständen beruhen. Man denke hier zum Beispiel an den Service DeepL/Linguee für KI-gestützte Sprachübersetzungen, der als (kostenfrei verfügbarer) Benchmark betrachtet werden kann. Weiterhin ist festzulegen, wie KI-Dienste abgegrechnet werden können, z.B. pro Abruf an einer Datenschnittstelle, pro entwickeltem und eingesetzen KI-Modell, o.ä. Schliesslich muss geklärt werden, wie die Bepreisung unterschiedlicher Dienstleister verglichen werden kann und welche Einkaufsstrategien, zum Beispiel durch Beschaffung von Nutzungskontingenten, erfolgsversprechend sind.

\subsection{Rechtliche Gestaltungsfragen}

Das rechtliche Handlungsfeld umfasst sämtliche Aspekte rund um den Schutz des geistigen Eigentums (inkl. Urheberrecht), dem Umgang mit Verbindlichkeiten aus KI-gestützten Systemen und dem Verständnis von Verantwortlichkeit in diesem Kontext. Für den produktiven Einsatz von KI ergeben sich mindestens zwei Aufgabenstellungen:

Erstens, die Sicherung von geistigem Eigentum (eng. Intellectual Property) an KI-Modellen. Die Parameter in KI-Modellen werden durch Training ständig verändert. Bei der Portierung von KI-Modellen (und ggf. nachfolgendem Transferlernen) in andere Kontexte ist der Veränderungsgrad zum ursprünglichen Modell nur begrenzt messbar. Insbesondere in der Zusammenarbeit mit Drittparteien (z.B. Outsourcing, Nutzung von APIs, oder der Zusammenarbeit mit Technologieanbietern und Start-Ups) stellen sich unterschiedliche Fragestellungen in Hinblick auf die Sicherung von IP an KI-Modellen. Insbesondere muss jederzeit sichergestellt sein, wer an einem KI-System welche Rechte besitzt. Weiterhin gilt es sicherzustellen, dass Unternehmen für erfolgskritische Anwendungen oder Produkte die Kontrolle über geistiges Eigentum behalten. Hierbei ist auch zu klären wie Verträge mit Drittparteien entsprechend gestaltet werden müssen. Die Schaffung von Transparenz zu den Urheberrechten ist ein zentrale Aufgabe im Management von betrieblichen KI-Systemen.

Zweitens, die Gestaltung haftungsrechtlicher Fragestellungen, die sich aus dem Betrieb bzw. Inverkehrbringen von KI-Systemen ergeben. Im heutigen Zeitalter der Netzwerk- und 
Plattform-Ökonomie werden in der Umsetzung von KI-Projekten oftmals eine Reihe unterschiedlicher, aber hochspezialisierter Dienstleister und Systeme orchestriert um eine KILösung aus unterschiedlichen Komponenten und Diensten zusammenzustellen. Dementsprechend ist es wichtig zu klären, welche Risiken der Betrieb eines KI-Systems mit sich bringt und vor allem welcher Akteur bei Eintreten bestimmter Ereignisse für entstandene Schäden haftbar ist. Dies gilt zum Beispiel für die Absicherung gegenüber Risiken im Zusammenhang mit automatisierten Fahrzeugen, der missbräuchlichen Nutzung von Daten durch Drittparteien, oder auch für fehlerhafte, durch KI-Algorithmen getroffene Entscheidungen, z.B. in medizinisch-diagnostischen Anwendungen.

\subsection{Sicherstellung von Compliance mit Regulierungen}

In diesem Handlungsfeld liegt der Fokus auf neuen Anforderungen von Regulierungsbehörden, die gegebenenfalls in einer neuen Governance für KI-Systeme implementiert werden müssen. Da KI-Systeme nicht deterministisch sind können sich die generierten Vorhersagen und Entscheidungen nach dem Lernen eines Modells verändern. Dementsprechend wird der Einsatz von KI-gestützten Entscheidungssystemen, insbesondere in sicherheitsrelevanten Industrien von Regulierungsbehörden mindestens kritisch geprüft und in naher Zukunft mit entsprechenden Regulierungsinstrumenten adressiert werden. Die Anwendungsbereiche sind zahlreich und betreffen neben Banken und Versicherungen, auch Maschinen, Medizinprodukte, Fahrzeuge, sowie auch das Management interner Prozesse und digitaler Kundeninteraktionen. Es ergeben sich zwei konkrete Aufgabenstellungen:

Erstens, Identifikation, Verständnis und Übersetzung der anwendbaren regulatorischen Anforderungen auf den Lebenszyklus von KI-Systemen. Unternehmen müssen sich mit den für ihre Industrie geltenden regulatorischen Anforderungen vertraut machen. Hierbei gilt es die Position entsprechender Regulierer und deren Instrumente zu verstehen und Massnahmen abzuleiten, um die entsprechende Compliance sicherzustellen. Diese können zum Beispiel auf typische, übergeordnete regulatorische Zielsetzungen abzielen, wie zum Beispiel das dokumentierte Testen IT-Systemen für die nachweisliche Sicherung von Patientensicherheit bei medizinischen Systemen und Produkten, die Nachvollziehbarkeit von finanziellen Entscheidungen um Solvenz, Transparenz oder allgemein auch finanzielle Stabilität sicherzustellen.

Zweitens, Implementierung von Governance, um diese Regularien umzusetzen. Hierzu gehört die Entwicklung und Anwendung entsprechender Compliance-Richtlinien und Wahl der geeigneten Nachweise. Hierzu zählen zum Beispiel Prozesse für die Implementierung und das Testen von KI-Systemen, Verfahren der Risikobewertung, sowie der Wahl einer geeigneten Dokumentation von KI-Systemen. Dieser Teil des Managements von KI ist eng mit der Versionierung und dem Umgang mit Cybersicherheits-Risiken, sowie Verzerrungen in Trainingsdaten (eng. Data Bias) verbunden. 


\subsection{Management des KI-Lebenszyklus}

Das Management des KI-Lebenszyklus ist das operative Bindeglied zwischen der betrieblichen Leistungserstellung und der eingesetzten KI-Technologie. Diese Perspektive fokussiert auf die Entwicklung von KI-Prototypen und produktiven KI-Lösungen und beinhaltet sowohl technische Aspekte, wie z.B. Datenakquisition, -exploration und KI-Modellentwicklung, als auch die Zuordnung relevanter Rollen, häufig z.B. Product Owner, Data Engineer, KISpezialisten oder Data Scientist. Als Bindeglied zwischen KI-bezogenen geschäftlichen und technologischen Fragestellungen schliesst das Lebenszyklus-Management sämtliche Phasen der KI-Entwicklung ein. Hierzu zählen zum Beispiel Ideengenerierung, Spezifizierung der zu lösenden Aufgabe, Akquisition von Trainingsdaten, Modellentwicklung, Modellevaluation und Release-Management (Miao, Li, Davis, \& Deshpande, 2017). Das Management dieser Phasen ist für einen reibungslosen Übergang vom KI-Prototypen in den Produktivbetrieb unerlässlich und beinhaltet neben der eigentlichen Modellentwicklung mindestens zwei weitere zentrale Aufgabenstellungen:

Erstens, das Management von Trainingsdaten und damit eng verbunden das Handling von Verzerrungen in Daten (eng. Data Bias). Es hat sich in bereits vielen Fällen gezeigt, dass die unzureichende Zusammenstellung von Trainingsdatensets zu sowohl technisch fehlerhaften, als auch zu sozial inakzeptablen Ergebnissen führen kann. Beispiele hierfür sind z.B. manipulierte Verkehrsschilder, die eine Fehlbewertung von KI-Modellen für das autonome Fahren provozieren, oder auch ein Seifenspender, der mit Verfahren der Bilderkennung nur bestimmte ethnische Gruppen bedient hat. Wesentliche Eigenschaften von KI-Modellen werden durch die ausgewählten Trainingsdaten definiert. Wesentliche Eigenschaften von KIModellen werden durch die ausgewählten Trainingsdaten definiert. Das Management von Trainigsdaten schliesst also die Entwicklung von Kriterien und Prozessen ein, die es erlauben, einen Bias in Trainingsdaten zu erkennen, zu prüfen und Strategien für das Ergreifen von Gegenmassnahmen zu definieren. Der Umgang mit Vorurteilen in Trainingsdaten ist ein wichtiger Teil des Risikomanagements von KI-Systemen.

Zweitens, die Versionierung und das Versionsmanagement von KI-Modellen. KI-Modelle besitzen häufig Millionen von gewichteten Parametern, die sich durch Training kontinuierlich verändern. Beim Training handelt es sich um nicht-konvexe Optimierungsprobleme. D.h. ein globales Optimum von Modell-Parametern kann nicht garantiert werden, so dass KI-Modelle mit jedem Training (oder Transferlernen) deutlichen Veränderungen unterworfen sein können. Bislang gibt es jedoch nur wenig entwickelte Ansätze, um Modellveränderungen nachzuvollziehen und z.B. durch Ähnlichkeitsmasse abzubilden (Miao, Li, Davis, \& Deshpande, 2017). Im Rahmen des KI- Lebenszyklus-Management ist es deshalb wichtig, ein effektives Versionsmanagement zu entwickeln, in dem Rollen, Strukturen, Prozesse und Software-Tools definiert sind, welche das kontrollierte Verändern von KI-Modellen ermöglichen. Die einwandfreie Beherrschung des Versionsmanagements von KI-Modellen ist 
insbesondere im Zusammenhang mit den vorgenannten Compliance, Haftungs- und Dokumentationsfragestellungen unmittelbar relevant.

\subsection{KI-Technologie-Infrastruktur}

Dieses Handlungsfeld bearbeitet Fragestellungen rund um Auswahl, Aufbau und Bereitstellung der notwendigen KI-Technologie-Infrastruktur. Hierbei sind mehrere Aufgaben zu berücksichtigen:

Erstens, die Auswahl geeigneter KI-Frameworks, KI-Bibliotheken und KI-Plattformen, damit KI-Modelle gemeinsam entwickelt und auch innerhalb der Organisation geteilt werden können. Hierbei gilt es Rahmenbedingungen zu schaffen, die es KI-Experten ermöglichen, die für den jeweiligen Einsatzzweck geeigneten Tools effektiv einzusetzen. In der praktischen Umsetzung hat sich gezeigt, dass Präferenzen entsprechender Teams hinsichtlich der eingesetzten und beherrschten Tools nicht unerheblich für die erfolgreiche KI-ModellEntwicklung sind. Daneben sind schliesslich auch ökonomische Aspekte zu berücksichtigen. Wie angeführt benötigt das Training von KI-Modellen grosse Rechenkapazitäten, zum Beispiel um die Parameter eines Neuronalen Netzes zu berechnen. Sobald das Training allerdings abgeschlossen und ein Modell produktiv geschaltet wird, wird Rechenleistung nur noch in viel geringerem Umfang benötigt und kann auch auf andere (CPU-basierter) Hardware durchgeführt werden. Vor diesem Hintergrund gilt es also die Computing-Infrastruktur so aufzubauen, dass sich diese kosteneffizient an die anfallenden Bedarfe skalieren lässt. Diese Ressourcenbündelung (eng. Ressource-Pooling) und daraus resultierende Skalierbarkeit der technologischen Infrastruktur ist einer der wesentlichen Vorteile, den Cloudanbieter ihren Kunden heute liefern können.

Zweitens, die Weiterentwicklung bestehender IT-Infrastrukturprozesse. Die Professionalisierung der IT ist in den meisten grösseren Unternehmen bereits sehr weit fortgeschritten. So finden sich in den meisten IT-Organisationen heute selbstverständlich gut beherrschte Prozesse, z.B. für das IT-Service Management, welches klare Vorgaben für das IT-Change- und Release Management liefert und auch den Betrieb und die Administration der IT-Infrastruktur regelt. Die Integration von neuer KI-Hard- und Software in solche bestehenden, häufig historisch gewachsenen IT-Infrastrukturen ist dementsprechend eine weitere wichtige Aufgabe für den betrieblichen Einsatz von KI, um den kontrollierten und sicheren Betrieb von KI-Systemen zu ermöglichen.

\subsection{Cybersicherheit von KI-Systemen}

Wie dargestellt ergeben sich beim Einsatz von KI neuartige Möglichkeiten für gegnerische Angriffe durch Manipulationen an der Datenschnittstellt. Dementsprechend zielt dieses Handlungsfeld auf Cybersicherheit im Kontext von KI-Systemen ab. Es sind mindestens zwei Aufgaben zu bearbeiten: 
Erstens, die systematische Identifikation von Angriffspunkten auf eingesetzte KI-Systeme. Im ersten Schritt gilt es, die eingesetzten KI-Systeme im Hinblick auf Ihre Angreifbarkeit durch Manipulationen an der Datenschnittstelle zu bewerten. KI-Systeme, insbesondere solche die per Design, mit einer eher unkontrollierten Umgebung interagieren, wie z.B. autonome Fahrzeuge, Roboter oder über offene Applikationsschnittstellen (eng. Open APIs), sind besonders anfällig für den vorgenannten Typ gegnerischer Angriffe. Im Zielbild braucht es im Managementsystem definierte Security-Prozesse und -Werkzeuge, mit denen die auftretenden Risiken eingeschätzt und minimiert werden können. Hierzu zählt zum Beispiel das aktive beobachten von Anfragen an Datenschnittstellen von KI-Systemen in Verbindung mit entsprechenden Analysen um Anomalien oder Manipulationen erkennen zu können.

Zweitens, das Bewusstsein in der Belegschaft darüber schaffen, dass es im Umgang mit KI neue Bedrohungsszenarien gibt. Neben technischen Massnahmen ist es von grosser Bedeutung, dass die in KI-Systeme involvierten Mitarbeiter hinreichend sensibilisiert für diese neuartigen Bedrohungen sind und auf etwaige Manipulationen oder Angriffe angemessen reagieren können.

\section{Zusammenfassung und Ausblick: Die nächsten Schritte mit KI}

In diesem Artikel wurden unterschiedliche Herausforderungen für das Management von Künstlicher Intelligenz im produktiven, betrieblichen Einsatz beschrieben. Das vorgestellte St.Galler Management Modell für KI (SGMM-KI) verortet insgesamt sieben KI-bezogene Handlungsbereiche: Management, Organisation, Recht, Compliance, Lebenszyklus, Technologie-Infrastruktur und Cybersicherheit. Mit dem vorgetellten SGMM-KI liegt somit ein Entwurf für ein Managementsystem vor, das einen Überblick über die Chancen und Herausforderungen gibt, die im Management von KI liegen.

Die Aufteilung in geschäftliche und technologische Handlungsbereiche berücksichtigt dementsprechend auch die heute vorzufindende betrieblichen Praxis, denn in vielen Unternehmen stehen sich zwei Lager gegenüber. Auf der einen Seite stehen KI-Experten und technologieaffine Mitarbeiter (häufig in der IT). Diese sind meist technisch aufgestellt, entweder durch Ausbildung oder kontinuierliche Weiterbildung, sie besitzten ein gutes bis Expertenverständnis von KI-Algorithmen und einzusetzenden Verfahren. Der Fokus dieser Gruppe besteht häufig darin KI-Technologie und KI-Verfahren zu überschauen und zielgerichtet einzusetzen. Auf der anderen Seite stehen Mitarbeiter, die in direkten Wertschöpfungsfunnktionen bzw. Unterstützungsfunktionen tätig sind. Viele dieser Mitarbeiter sehen KI entweder als grosse Chance, häufig aber auch als grosses persönliches und unternehmerisches Risiko, dem auch mit Angst, Sorge oder Ablehnung begegenet wird.

Im Hinblick auf diesen noch jungen Einsatz von KI in Unternehmen möchten die Autoren dem geneigten Leser dann auch zwei konkrete Empfehlungen mit auf den Weg geben, die neben 
der

Beherrschung der aufgezeigten Managementaufgaben

als

konkrete

Führungsherausforderungen betrachtet werden sollten:

Zum einen sollten sich Führungskräfte aktiv in den Abbau von Vorbehalten gegenüber KI einbringen. Im Austausch mit CIOs und Unternehmern, aber auch im Rahmen einer durch das Institut für Wirtschaftsinformatik der Universität St.Gallen durchgeführten quantitativen Befragung von KI-Anwendern und Nicht-Anwendern wurde sehr deutlich, dass die grösste Hürde für die Einführung von KI nicht so sehr in der Auswahl geeigneter algorithmischer Verfahren liegt, sondern vielmehr in der Bereitstellung von Daten sowie der Akzeptanz der zu etablierenden KI-Lösung durch Mitarbeiter und Kunden. Führungskräfte sind dementsprechend gut beraten (wenigstens) einführende Weiterbildungsmöglichkeiten zu etablieren, damit sich interessierte Mitarbeiter über Einsatzmöglichkeiten, Nutzen und Funktionsweise, aber auch über Risiken im Zusammenhang von KI informieren können. Darüber hinaus ist es wichtig KI-Systeme im Einklang mit in der Unternehmenskultur akzeptieren Praktiken, z.B. bezogen auf das Teilen von Daten, der Wertschätzung von Domänenexperten, usw. zu implementieren. Entwicklungsansätze wie das Human-Centered Design bieten hierfür beispielsweise ein entsprechendes methodisches Reportoire.

Zum anderen wird eine wichtige Herausforderung darin bestehen KI systematisch in die betriebliche Breite $\mathrm{zu}$ skalieren. Hierzu ist es erforderlich von einer ambitionierten Prototypenentwicklung $\mathrm{zu}$ einer ambitionierten produktiven Skalierungsstrategie hinzuarbeiten, welche die organisatorische Umsetzung von KI-Modellen aus dem Testlabor in die produktive Nutzung beschreibt. Hierbei spielt insbesondere auch die nachhaltige Organisation des Skalierungsprozesses eine wichtige Rolle, so dass sowohl KI-Lösungen effektiv entwickelt werden, aber auch Good/Best-Practices und Kompetenzen in weitere Geschäftseinheiten und Anwendungskontext transferiert werden können.

Das zukünftige Management von produktiven KI-Anwendungen in der betrieblichen Praxis bleibt spannend. Obgleich das Thema derzeit eine hohe mediale Aufmerksamkeit geniesst, wird sich noch zeigen müssen wie gut es Unternehmen gelingt die ensprechenden Einstellungen, Fähigkeiten und Prozesse zu realisieren, die nötig sind, um das grosse „Versprechen“ Künstliche Intelligenz systematisch in der betrieblichen Wertschöpfung einsetzen zu können. 


\section{Literatur}

Conboy, K. (2009). Agility from first principles: Reconstructing the concept of agility in information systems development. Information Systems Research, 20(3), 329-354.

European Commission. (2019, April 8). Ethics guidelines for trustworthy AI [Text]. Retrieved December 6, 2019, from European Commission website: https://ec.europa.eu/digital-singlemarket/en/news/ethics-guidelines-trustworthy-ai

Goodfellow, I. J., Shlens, J., \& Szegedy, C. (2014). Explaining and harnessing adversarial examples. ArXiv Preprint ArXiv:1412.6572.

Goodin, D. (2019, April 2). Researchers trick Tesla Autopilot into steering into oncoming traffic. Retrieved December 6, 2019, from Ars Technica website: https://arstechnica.com/information-technology/2019/04/researchers-trick-tesla-autopilotinto-steering-into-oncoming-traffic/

Handelsblatt. (2019, March 13). ZF beschleunigt bei Künstlicher Intelligenz die Aufholjagd auf Bosch. Retrieved December 6, 2019, from https://www.handelsblatt.com/unternehmen/industrie/autozulieferer-zf-beschleunigt-beikuenstlicher-intelligenz-die-aufholjagd-auf-bosch/24099472.html

LeCun, Y., Bengio, Y., \& Hinton, G. (2015). Deep learning. Nature, 521(7553), 436-444.

Miao, H., Li, A., Davis, L. S., \& Deshpande, A. (2017). Modelhub: Deep learning lifecycle management. 1393-1394. IEEE.

Reuters. (2018, October 10). Amazon scraps secret AI recruiting tool that showed bias against women. Reuters. Retrieved from https://uk.reuters.com/article/us-amazon-com-jobsautomation-insight-idUKKCN1MK08G

von Krogh, G. (2018). Artificial intelligence in organizations: New opportunities for phenomenon-based theorizing. Academy of Management Discoveries.

Wang, X., Conboy, K., \& Pikkarainen, M. (2012). Assimilation of agile practices in use. Information Systems Journal, 22(6), 435-455.

Zhang, M. (2015). Google Photos Tags Two African-Americans As Gorillas Through Facial Recognition Software. Retrieved December 6, 2019, from Forbes website: https://www.forbes.com/sites/mzhang/2015/07/01/google-photos-tags-two-africanamericans-as-gorillas-through-facial-recognition-software/ 
7. Anhang 1: Das St.Galler Management Modell für den betrieblichen Einsatz von Künstlicher Intelligenz

Management von Künstlicher Intelligenz (KI)

(1) Initiale KI-Projekte durchführen, (2) Organisationales Lemen sicherstellen

Organisation
des Betriebs
(1) Identifikation von
KI-Potentialen in
Business und Use
Cases
(2) Aufbau und
Entwicklung von KI-
Fähigkeiten in der
Organisation
(3) Gestaltung des
Anbietermanagements
für KI-Dienste

Rechtliche

Gestaltung

(1) Sicherung von geistigem Eigentum an

KI-Modellen

(2) Gestaltung

haftungsrechtlicher

Fragestellungen

für KI-Dienste
Regulierung \& Compliance

(1) Identifikation und

Übersetzung der

anwendbaren

regulatorischen

Anforderungen

(2) Implementierung

von Governance, um

Regularien

umzusetzen

\section{Lebenszyklus- Management}

(1) Management von Trainingsdaten und

Data Bias

(2) Versionierung und Versionsmanagement von $\mathrm{KI}$-Modellen

\section{Technologie-} Infrastrukur

(1) Auswahl

geeigneter KI-

Frameworks, $\mathrm{KI}-$

Bibliotheken und KI-

Plattformen

(2) Weiterentwicklung

bestehender IT-

Infrastrukturprozesse
Cyber-

sicherheit

(1) Identifikation von Angriffspunkten auf

eingesetzte KI-

Systeme

(2) Bewusstsein für neue KI-Bedrohungsszenarian schaffen 